\title{
A STELLAR TIDAL STREAM AROUND THE WHALE GALAXY, NGC 4631
}

\author{
David Martínez-Delgado ${ }^{1}$, Elena D’Onghia ${ }^{2,7}$, Taylor S. Chonis ${ }^{3}$, Rachael L. Beaton ${ }^{4}$, Karel Teuwen ${ }^{5}$, \\ R. Jay GaBany ${ }^{6}$, Eva K. Grebel ${ }^{1}$, and Gustavo Morales ${ }^{1}$ \\ ${ }^{1}$ Astronomisches Rechen-Institut, Zentrum für Astronomie der Universität Heidelberg, Mönchhofstr. 12-14, D-69120 Heidelberg, Germany \\ ${ }^{2}$ University of Wisconsin, 475 N. Charter St., Madison, WI 53706, USA \\ ${ }^{3}$ Department of Astronomy, University of Texas at Austin, 2515 Speedway, Stop C1400, Austin, TX 78712, USA \\ ${ }^{4}$ Observatories of the Carnegie Institution of Washington, 813 Santa Barbara Street, Pasadena, CA 91101, USA \\ ${ }^{5}$ Remote Observatories Southern Alpes, Verclause, France \\ ${ }^{6}$ Black Bird Observatory II, Alder Springs, CA, USA \\ Received 2014 October 23; accepted 2015 June 29; published 2015 September 18
}

\begin{abstract}
We report the discovery of a giant stellar tidal stream in the halo of NGC 4631, a nearby edge-on spiral galaxy interacting with the spiral NGC 4656, in deep images taken with a $40 \mathrm{~cm}$ aperture robotic telescope. The stream has two components: a bridge-like feature extending between NGC 4631 and NGC 4656 (stream ${ }_{\mathrm{SE}}$ ) and an overdensity with extended features on the opposite side of the NGC 4631 disk ( stream $_{\mathrm{NW}}$ ). Together, these features extend more than $85 \mathrm{kpc}$ in projection. The orientation of stream $\mathrm{SE}_{\mathrm{SE}}$ relative to the orientations of NGC 4631 and NGC 4656 is not consistent with an origin from an interaction between these two spirals, and is more likely debris from a satellite encounter. The stellar tidal features can be qualitatively reproduced in an $N$-body model of the tidal disruption of a single, massive dwarf satellite on a moderately eccentric orbit $(e=0.6)$ around NGC 4631 over $\sim 3.5$ Gyr. Both modeling and inferences from the morphology of the streams indicate these are not associated with the complex HI tidal features observed between both spirals, which likely originate from a more recent, gas-rich accretion event. The structure of stream ${ }_{\mathrm{NW}}$ suggests that it may contain the progenitor of the stream, in agreement with the $\mathrm{N}$-body model. However, we cannot exclude other possibilities such as the satellite dwarf galaxy NGC 4627 being the progenitor based on these data. In addition, stream $\mathrm{NW}_{\mathrm{N}}$ is roughly aligned with two very faint dwarf spheroidal candidates. The system of dwarf galaxies and the tidal stream around NGC 4631 can provide an additional interesting case for exploring the anisotropy distribution of satellite galaxies recently reported around Local Group spiral galaxies by means of future follow-up observations.
\end{abstract}

Key words: galaxies: dwarf - galaxies: evolution - galaxies: photometry - Local Group

\section{INTRODUCTION}

Numerical cosmological models built within the $\Lambda$-cold dark matter $(\Lambda$-CDM) paradigm (Bullock \& Johnston 2005; Cooper et al. 2010; Pillepich et al. 2014) predict that deep imaging of the stellar halos of nearby large spiral galaxies should display a wide variety of diffuse, low-surface brightness stellar substructures. The most common features are stellar streams or shells, which result from interactions between the parent galaxy and its dwarf satellite companions. As implied by the $\Lambda$-CDM paradigm, the existence of such features is a ubiquitous feature of all galaxies, although this remains untested observationally. This is largely due to the challenge of producing images with sufficient surface brightness sensitivity and low background fluctuations to reliably detect such faint features. Although more luminous examples of diffuse stellar streams and shells around massive elliptical galaxies have been known for many decades (e.g., Arp 1966; Schweizer \& Seitzer 1988; Duc et al. 2014), recent studies have detected much fainter analogues of these structures around spiral galaxies in the local universe, including the Milky Way (MW) and Andromeda. Moreover, recent extensive photometric databases have for the first time provided spectacular panoramic views of the MW tidal streams (Majewski et al. 2003; Belokurov et al. 2006; Slater et al. 2014) and revealed the existence of large stellar sub-structures within the MW halo, which have been interpreted as observational evidence to understand our

\footnotetext{
${ }^{7}$ Alfred P. Sloan Fellow.
}

Galaxy's past and present hierarchical formation. Likewise, the Pan-Andromeda Archaeological Survey (McConnachie et al. 2009) has provided a panoramic view of the Andromeda halo, which reveals a striking diversity of halo sub-structures akin to those observed in the MW. These observations provide sound empirical support for the $\Lambda$-CDM prediction that tidally disrupted dwarf galaxies are important contributors to stellar halo formation, at least for the Local Group spirals.

While stellar streams in the MW and Andromeda can be studied in detail from studies using resolved stellar populations, direct comparison of these features to cosmological models is complicated by "cosmic variance" - the fact that each large spiral has experienced a unique hierarchical formation history that depends on the number and timing of satellite accretions, as well as both the internal kinematics and orbital properties of the individual satellites. A search for analogues to those features observed in the MW and Andromeda across a larger sample of nearby spiral galaxies is required to understand if the recent merger histories for Local Group spirals are "typical," an issue that remains unclear (Mutch et al. 2011). Current $\Lambda$-CDM numerical simulations can guide this quest for star-stream observational signatures (e.g., Johnston et al. 2008; Cooper et al. 2010). These simulations have demonstrated that the characteristics of sub-structure currently visible in stellar halos are sensitive to recent $(0-8 \mathrm{Gyr}$ ago $)$ merger events of galaxies, which is a timescale that corresponds to the last few tens of percent of mass accretion for a spiral galaxy like the MW. These models predict that a survey of $\sim 100$ parent galaxies reaching a surface brightness of $\sim 29 \mathrm{mag} \operatorname{arcsec}^{-2}$ would reveal 
many tens of tidal features, perhaps nearly one detectable stream per galaxy (at this surface brightness limit). A direct comparison of these simulations with actual observations is not possible because no suitable data set yet exists. Thus, the observational portrait of satellite accretion events is far from complete.

Recent deep, wide-field images of nearby MW analogue galaxies within the Local Volume taken with small telescopes have revealed an assortment of large-scale tidal structures in the halos of several nearby galaxies. ${ }^{8}$ For the first time, the detected features exhibit the striking morphological diversity predicted by cosmological models (Martínez-Delgado et al. 2010; MD2010). In addition, a systematic automated search around 450 spiral galaxies in the Sloan Digital Sky Survey (SDSS) has provided the first estimate of the frequency of tidal features around spiral galaxies to a limiting surface brightness of $27-28 \mathrm{mag} \operatorname{arcsec}^{-2} \quad(\sim 10 \%-15 \%$; Miskolczi et al. 2011). Both observational projects have also yielded an unprecedented sample of bright stellar streams in nearby spiral galaxies, including the first discovery of observational analogues to the canonical morphologies predicted from $N$ body models of stellar halos that are entirely constructed from satellite accretion (Johnston et al. 2008). In addition, there are other ongoing projects to map the surroundings of nearby massive galaxies to detect tidal streams and new satellites (e.g., Ludwig et al. 2012; Duc et al. 2014; Abraham \& van Dokkum 2014). The detection of these structures offers a unique opportunity to study the still dramatic last stages of galaxy assembly in the local universe (e.g., Foster et al. 2014) and to compare the frequency and detailed characteristics (e.g., surface brightness distribution, morphology, proportion of stellar luminosity in the host's smooth halo versus level of substructure) of tidal stellar features against those predicted for MW-sized galaxies in the $\Lambda$-CDM paradigm.

Encouraged by these results, the Stellar Tidal Stream Survey (PI. Martínez-Delgado) is carrying out the first systematic survey of stellar tidal streams to a surface brightness sensitivity of $\mu_{r}=28.5 \mathrm{mag} \operatorname{arcsec}^{-2}$ using a network of small, robotic telescopes placed on different continents. In this paper, we report the discovery of a giant stellar stream in one of the classical and extensively studied galaxies, NGC 4631 (7.4 $\pm 0.1 \mathrm{Mpc}$; Radburn-Smith et al. 2011), also known as the "Whale galaxy." These diffuse light features have also independently discovered by Karachentsev et al. (2014). Imaging observations and data reduction are described in Section 2. The stream is described and placed in the context of other sub-structure in NGC 4631 in Section 3. The stream properties are measured in Section 4 and used to construct a suitable $N$-body model in Section 5 . The work is summarized and discussed in Section 6.

\section{OBSERVATIONS AND DATA REDUCTION}

Deep imaging of the field around NGC 4631 was collected at the Remote Observatory Southern Alps (ROSA, Verclause, France) with a $40 \mathrm{~cm}$ aperture $f / 3.75$ corrected Newtonian telescope. A FLI ML16803 CCD camera was used and provided a pixel scale of $1^{\prime \prime} .237$ pixels $^{-1}$ over a $81^{\prime} \times 81^{\prime}$ field of view. A set of individual $300 \mathrm{~s}$ images were obtained

\footnotetext{
8 These MW and M31 surveys mentioned above use resolved star counts to probe effective surface brightness levels several magnitudes fainter than is possible in these small aperture telescope surveys.
}

remotely with an Astrodon Gen2 Tru-Balance E-series luminance filter over several photometric nights between 2011 December and 2012 February. As shown in Figure 1(a), this luminance filter is a wide-band, nearly tophat filter that transmits from $400 \lesssim \lambda(\mathrm{nm}) \lesssim 700$, and broadly covers the more familiar $g$ and $r$ bands utilized by the SDSS. Each individual exposure was reduced following standard image processing procedures for dark subtraction, bias correction, and flat fielding adopted for the larger stream survey (MD2010). The images were combined to create a final coadded image with a total exposure time of 23,100 s.

The luminance data were internally flux calibrated by utilizing SDSS Data Release 7 (DR7; Abazajian et al. 2009) $g$ and $r$-band photometry as described in the following procedure. First, we remove any residual large-scale sky gradients from our wide-field images by modeling the background with a two-dimensional, fourth-order Legendre polynomial. The polynomial was fit to the median flux within coarse spatial bins that were $5^{\prime}$ on a side after all $\geqslant 5 \sigma$ sources were masked. Aperture photometry was then performed on 27 isolated stars that were spatially distributed across the background-subtracted luminance image, and matched to the SDSS DR7 point source catalog. For each of these stars, the luminance magnitude $L$ is calculated from the measured stellar flux $f_{\mathrm{L}}$ as:

$$
L=-2.5 \log f_{\mathrm{L}}+c_{1},
$$

where $c_{1}$ is the photometric zero-point. Using the retrieved SDSS $g$ and $r$ magnitudes for each star, we define the SDSScalibrated luminance magnitude as:

$$
L_{\mathrm{SDSS}}=c_{2} g+\left(1-c_{2}\right) r \text {, }
$$

where $c_{2}$ is a coefficient that weighs the two SDSS photometric bands and is effectively a color term that will depend primarily on the wavelength-dependent sky-to-detector throughput of the ROSA system relative to that of the SDSS system. The photometric coefficients $c_{1}$ and $c_{2}$ are determined for the ROSA Luminance image by minimizing $L-L_{\text {SDSS }}$ for the ensemble of stars. Figure $1(\mathrm{~b})$ shows $L-L_{\mathrm{SDSS}}$ after the minimization for the 27 calibration stars as a function of $g-r$ color, from which the values of the coefficients were determined to be $c_{1}=27.920 \pm 0.012$ and $c_{2}=0.297 \pm 0.016$. The standard deviation in $L-L_{\text {SDSS }}$ is $0.036 \mathrm{mag}$. Since $c_{2}<0.5$, the $L$ magnitudes measured from the ROSA luminance data are similar to the SDSS $r$-band. This is confirmed in Figure 1(c), in which the ROSA $L$ magnitudes using the aforementioned zeropoint are plotted as a function of SDSS $r$ magnitudes for the ensemble of 27 calibration stars. A linear fit to the data yields $L=1.011 \pm 0.006 r+0.069 \pm 0.094$. As can be seen in Figure $1(\mathrm{c})$, the scatter about this linear relation is a strong function of $g-r$ color. After subtracting the linear fit, the remaining $1 \sigma$ scatter is $0.138 \mathrm{mag}$ due to the uncorrected color term.

We use the method described in MD2010 for determining the limiting depth of our image, due to both photon noise and larger-scale background variations due to flat fielding errors, ghosts, and scattered light. To measure the photon noise limit, we calculate the mean of the standard deviation measured from 25 small $4 \times 4$ pixels boxes that were randomly distributed throughout the image away from stellar and extended sources. 

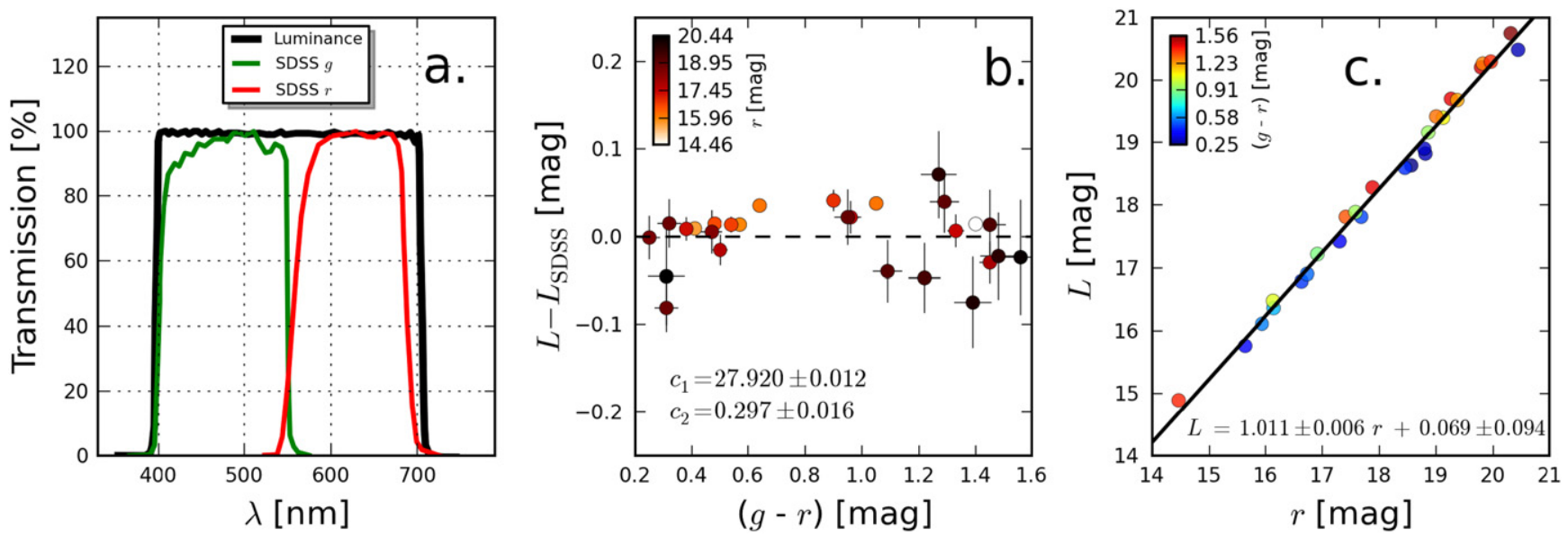

Figure 1. Calibration of the ROSA luminance photometry and comparison to the SDSS $g$ and $r$-bands. (a) Filter transmission curves for the wide-band luminance filter compared to those of the SDSS $g$ and $r$-bands, which are entirely contained within the pass-band of the luminance filter. (b) The residuals of the ROSA instrumental $L$ magnitudes and SDSS-derived $L$ magnitudes for the 27 calibration stars in the NGC 4631 field from the SDSS DR7 catalog. The data points are colored according to their SDSS $r$-band magnitude. The values of the derived photometric coefficients from Equations (1) and (2) that were used to calculate $L$ and $L$ SDSS, respectively, are also shown in the plot. These data are plotted as a function of $g-r$ color, and have a $1 \sigma$ spread 0.036 mag. (c) Using the zero-point $c_{1}$, the ROSA $L$ magnitudes are compared to the SDSS $r$-band magnitudes for the 27 calibration stars. This plot, and the associated linear fit shown by the black line, indicates that the $L$ magnitudes measured in the ROSA luminance image are very similar to SDSS $r$-band magnitudes. However, a color term correction is required to further reduce the $1 \sigma$ scatter about the linear relation $(0.138 \mathrm{mag})$, as indicated by the colored data points that correspond to the $g-r$ color of each SDSS calibration star.

To measure large-scale background fluctuations, we calculated five times the standard deviation of 25 randomly placed boxes in the background having a typical size comparable to the spatial extent of the faint features we intend to measure (here, $\sim 10 \operatorname{arcmin}^{2}$ in size). We find that the $5 \sigma$ limiting surface brightness of the ROSA luminance data for photon noise is $25.9 \mathrm{mag} \mathrm{arcsec}^{-2}$ while the $5 \sigma$ limit due to large-scale variations is $27.7 \mathrm{mag} \operatorname{arcsec}^{-2}$. As described in Chonis et al. (2011), the large-scale background variations are the dominant source of uncertainty for faint extended objects whose surface brightness approaches the latter measurement limit. For all surface brightness measurements that follow, the quoted uncertainty is calculated using a total error budget (including both random and systematic sources of error) that follows Section 2.3.4 of Chonis et al. (2011).

In our measurements, we correct for foreground Galactic extinction by adopting the value $A_{L}=c_{2} A_{g}+\left(1-c_{2}\right) A_{r}$. Using the extinction maps of Schlegel et al. (1998) together with an estimated extinction coefficient based on Schlafly \& Finkbeiner (2011), $A_{L}=0.044$ using the values of $A_{g}=0.056$ and $A_{r}=0.039$ at the coordinates of NGC 4631. Using this single value for the entire ROSA field of view is justified since the reddening in this area of the sky varies over only $0.015 \leqslant E(B-V) \leqslant 0.016$.

\section{THE STELLAR STREAM OF NGC 4631}

Our deep, wide field, panoramic view of the moderately interacting pair NGC 4631/4656 (with a projected separation of $R_{\mathrm{ps}}=69 \mathrm{kpc}$ ) is shown in Figure 2. Our deep image reveals two low surface brightness, extended structures on either side of NGC 4631. We will refer to the "bridge-like" feature to the southeast of NGC 4631 as stream $\mathrm{SE}$, and to that to the northwest as stream $_{\mathrm{NW}}$. These structures were also independently found by Karachentsev et al. (2014) in deep images obtained with amateur telescopes. In addition, the stream $_{\mathrm{NW}}$ is also visible in the POSS2 sky survey IIIaJ photographic plate of this region around NGC 4631. The two features are roughly aligned with each other at a position angle (PA) of $136^{\circ}$, which suggests a common origin. If so, the discontinuity of the structure on the NW side of the galaxy could be due to the limiting surface brightness of the ROSA image. Interestingly, stream $_{\mathrm{NW}}$ shows a more luminous inner region within the more elongated lower surface brightness feature. This structure is similar to that of a tidally disrupting dwarf galaxy (see, for example, NGC 4216 in MD2010; Figure 1 or Paudel et al. 2013) and suggests that it may be the progenitor of

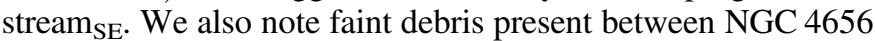
and its companion, named NGC 4656 UV by SchechtmanRook \& Hess (2012), indicative of interactions between these two galaxies. The detection of further, fainter structures is hampered by contamination from faint Galactic cirrus, which is barely visible in Figure 2.

Figure 3 shows a zoomed region with an appropriate size to fit the NGC 4631 stellar halo. For illustrative purposes, we have included a color inset of the disk of the galaxy taken with the Black Bird Observatory during the pilot survey of this project (MD2010). This better resolves the vertical structures of the disk and the morphological disturbances of NGC 4627, a dwarf Elliptical (dE) satellite of NGC 4631 clearly interacting with its parent galaxy. Although the extrapolation of the path of the stream $_{\mathrm{SE}}$ across the disk passes near the position of NGC 4627 in projection, the visible tidal arms of this companion are oriented almost perpendicular to the PA of the streams (see Figure 3). Since streams trace the paths of their progenitor system, this suggests that NGC 4627 is an unlikely candidate for the progenitor of stream $\mathrm{SE}$ and stream $_{\mathrm{NW}}$. Another possibility would be to assume that the stream $\mathrm{SE}_{\mathrm{SE}}$ is not related to the stream $\mathrm{NW}_{\mathrm{N}}$ and that NGC 4627 is the progenitor of both streams. Twisted inner isophotes due to tides often appear at completely different PAs as compared to the more extended tidal streams. However, the change in PAs seems to be very extreme in this case even compared to the case of the globular cluster Pal 5 (Odenkirchen et al. 2001, Figure 2), which shows a similar effect.

It is more likely that NGC 4627 is responsible for the optically detected extended vertical structure within the NGC 4631 disk 


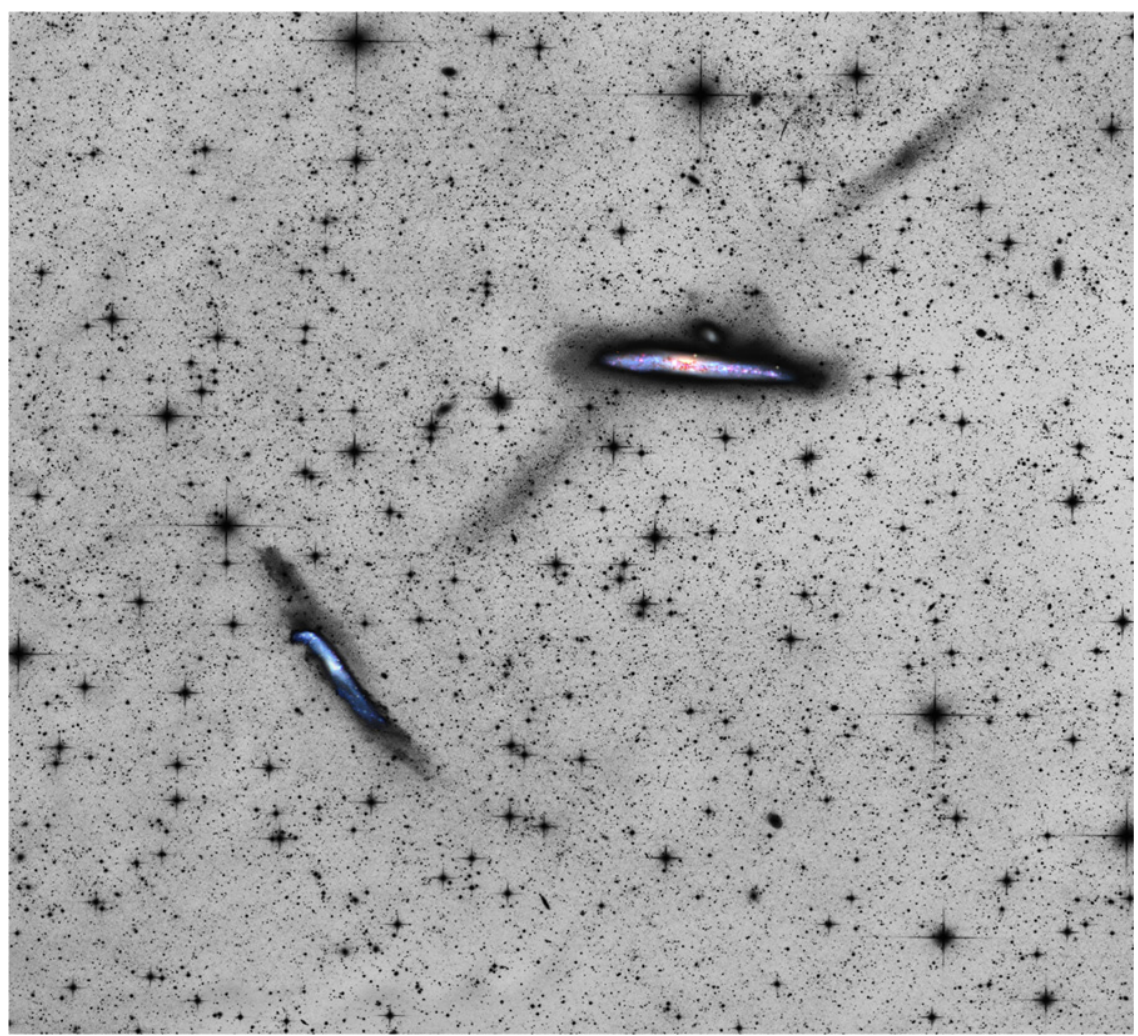

Figure 2. Final ROSA $L$ image of the NGC 4631 environs. The image is approximately $81^{\prime} \times 81^{\prime}$ and oriented such that east is to the left and north is at the top. NGC 4631 (the "Whale" galaxy) is the edge-on galaxy in the upper right. Its large companion is NGC 4656 to the lower left. The projected separation of the two galaxies is $\sim 32^{\prime}$ or $69 \mathrm{kpc}$. Several features are visible in the image, the most prominent being a stellar stream extending, in projection, from NGC 4631 toward

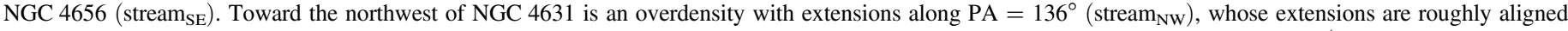
with the PA of stream $\mathrm{SE}$. We also note the detection of debris between NGC 4656 and its proposed tidal-dwarf companion, NGC 4656 UV (see Schechtman-Rook \& Hess 2012).

(Ann et al. 2011) or to the complex gas streams described below. Future observations like radial velocity measurements, deeper photometry or projected distances of different parts of the stream will be able to confirm or exclude NGC 4627 as progenitor of the "bridge-like" feature. In particular, color information would be essential.

An alternative explanation for the observed faint tidal features is that they could be due to the tidal tails from the interaction of NGC 4631 with its large-spiral companion, NGC 4656, a formation scenario that was also proposed to explain a similar extended plume in NGC 3628 (Rots 1978). However, as is discussed in Section 5, the edge-on perspective and visual configuration of both interacting systems and the morphology of the stream likely rules out this scenario.

Our deep image also detects the presence of two overdensities, marked as DW1 and DW2 in Figure 3. DW1, DW2, and the most luminous, inner portion of stream $_{\mathrm{NW}}$ were reported as dwarf companions of NGC 4631 by Karachentsev et al. (2014). The first smaller object, DW1, is well separated from NGC 4631 ( $\sim 10^{\prime}$ southeast; $\left.25 \mathrm{kpc}\right)$ and it could be a faint dwarf spheroidal satellite of NGC 4631. The second feature DW2, is located $5^{\prime}(\sim 12 \mathrm{kpc})$ north of the NGC 4631 core and its morphology is consistent with that of a faint, more extended dwarf galaxy satellite. Given the surface brightness limit of the luminance image, however, we cannot rule out a connection to the vertical structure of the NGC 4631.

In addition to the stellar streams and dwarf galaxy candidates already discussed, the NGC 4631 stellar halo contains a complex HI gas morphology. In fact, it contains two relatively unique features: (i) five individual $\mathrm{HI}$ tidal features extending from the disk (Rand \& van der Hulst 1993; Rand 1994); and (ii) two HI supershells associated with massive star forming regions that are expelling large masses of gas at high velocities (Rand \& van der Hulst 1993). Moreover, there is extensive diffuse $\mathrm{H} \alpha$ emission throughout the gas halo (Donahue et al. 1995), spatially coincident with the gaseous streams. We compare these data to investigate any potential associations between the stellar and gaseous substructures.

In Figure 4, we overlay the low-resolution HI zeroth order map of Rand (1994) on the ROSA luminance image. The HI maps were obtained with the Very Large Array (VLA) at a resolution of 45 ! $\times 89$ !. over a velocity range of $110-1023 \mathrm{~km}$ $\mathrm{s}^{-1}$ with velocity resolution of $10 \mathrm{~km} \mathrm{~s}^{-1}$. The single-channel point source sensitivity is $M_{\mathrm{HI}}=7 \times 10^{5} M_{\odot}$ for a source at the beam center. We refer the reader to Rand (1994) for additional details on the observations and data processing. The five tidal spurs as identified in the Rand (1994) data sets are labeled. Together, the spurs contain $3 \times 10^{9} M_{\odot}$ of HI gas, approximately one third of that measured for NGC 4631 and half of that measured for NGC 4656 (Rand 1994). Note that the stellar stream identified in Figure 2 is located between spurs 1 and 2. Six low-luminosity dwarf satellites of NGC 4631 are also labeled in Figure 4, including those identified from the HI mapping (Rand 1994) and those of Karachentsev et al. (2014).

Tidal spurs 1 and 4 are thought to have been formed by interactions between NGC4631 and its large galaxy 


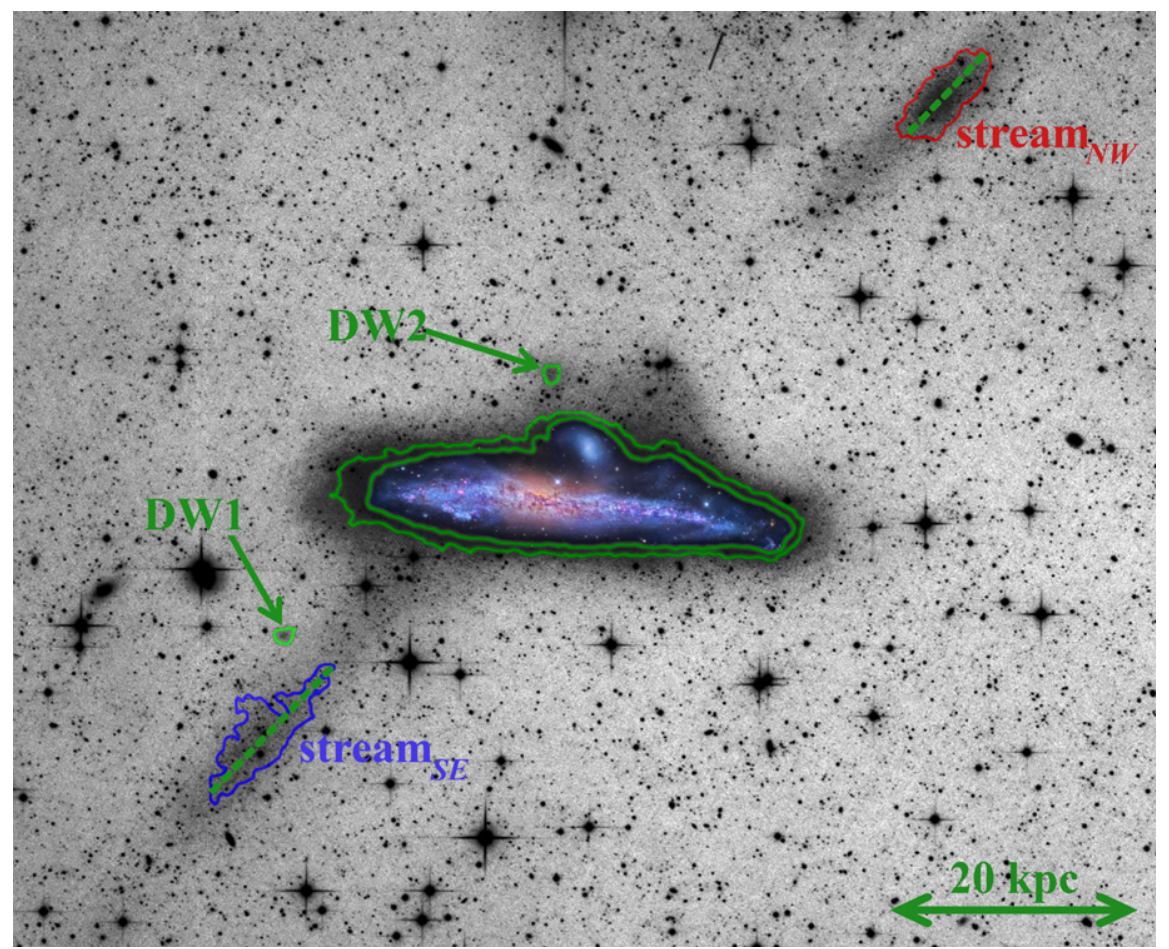

Figure 3. Zoom into the ROSA luminance image (Figure 1) to feature just the NGC 4631 stellar halo with isophotal boundaries overlaid on the major substructures. The image is approximately $42 ! 5 \times 34 ! 6$. The two green isophotes around NGC 4631 correspond to $\mu_{\mathrm{L}}=25 \mathrm{mag} \operatorname{arcsec}^{-2}$ and $\mu_{\mathrm{L}}=26 \mathrm{mag}^{\mathrm{arcsec}}{ }^{-2}$, respectively. The small, green isophote around DW2 corresponds to $\mu_{\mathrm{L}}=26.5 \mathrm{mag} \mathrm{arcsec}{ }^{-2}$, which is the faintest closed isophote that excludes emission from the NGC 4631 stellar halo. The green isophote for DW1, the red isophote for stream $\mathrm{NW}_{\text {and the blue isophote for the stream }}$ SE all correspond to $\mu_{\mathrm{L}}=27.7 \mathrm{mag} \operatorname{arcsec}^{-2}$, which is the $5 \sigma$ limiting surface brightness of the ROSA luminance data due to large-scale background variations. The green dashed lines superposed on the isophotes of stream $\mathrm{NW}$ and stream ${ }_{\mathrm{SE}}$ represent the major axes of the ellipses that were fit to each object's isophotal boundary.

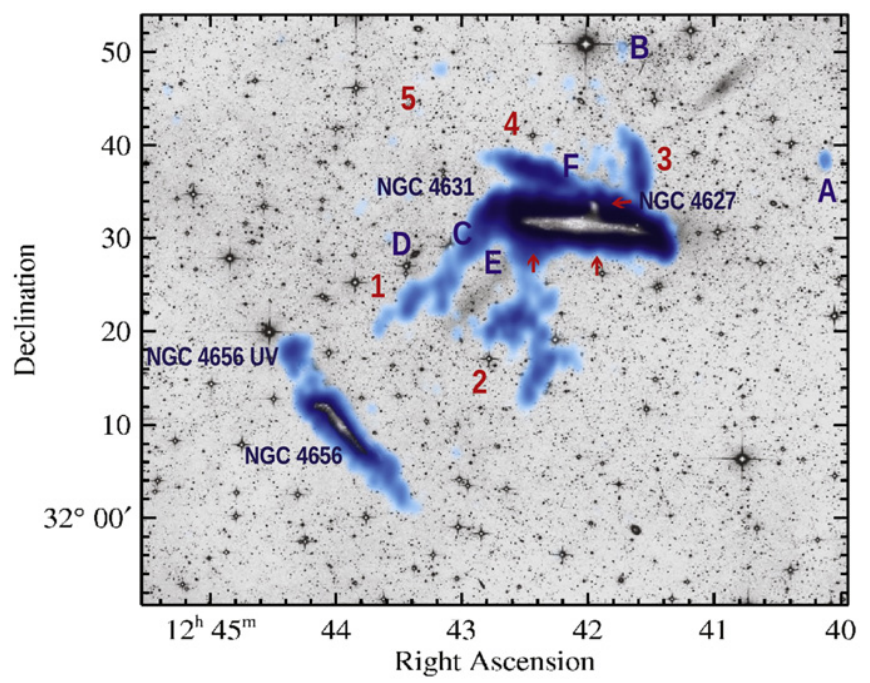

Figure 4. Distribution of neutral hydrogen in the NGC 4631 system (blue) overlaid on the ROSA Luminance image (Figure 1). The HI total intensities are taken from the VLA observations presented in Rand (1994) for the velocity range of $110-1023 \mathrm{~km} \mathrm{~s}^{-1}$. They are sensitive to a point source limit of $7 \times 10^{5}$ $M_{\odot}$. The five HI tidal features ("spurs") are labeled (1-5) and the two HI super-shells are indicated with arrows. Stream ${ }_{\mathrm{SE}}$ is juxtaposed between spurs 1 and 2 and is near a region of enhanced $\mathrm{H} \alpha$ emission (Donahue et al. 1995). No $\mathrm{HI}$ is associated with stream $\mathrm{NW}$. In addition to NGC 4627, there are six dwarf galaxies associated with NGC 4631, which are labeled in the diagram as follows-A: dwarf A (Rand 1994), B: Keeler 529, C: MCG+06-28-022, D: SDSS J124324.89+322854.2, E: DW3, and F: DW2. DW2 is embedded within Spur 4, but without a redshift, it cannot be directly associated with gas in the stream. companion, NGC 4656 (Combes 1978). However, an interaction between these two galaxies is unlikely to form gas streams around the higher mass object (NGC 4631, in our case), instead such tails tend to be found around the "victim" or the lower mass galaxies (here, NGC 4656). Spurs 2 and 3, on the other hand, were interpreted to have been formed by tidal interactions between NGC 4631 and NGC 4627, its tidally distorted, blue $\mathrm{dE}$ companion. Modeling of the tidal features, however, is complicated by the lack of reliable kinematic velocities for the dwarf companions in the field.

The projection of each of these features is interesting for many reasons. First, the stellar stream described in this paper is located in between the shells 1 and 2. Second, the stream intersects the disk (in projection) at one of the massive star forming regions. However, the stream is not of the morphology anticipated by the interaction of a gas rich galaxy of the mass needed to explain the gas streams, which are of similar mass as the Magellanic Stream around the MW $\left(3 \times 10^{9} M_{\odot}\right.$; Rand 1994). Gas-rich dwarf interactions tend to produce more concentrated stellar debris as, for example, the Great Southern Stream in the Andromeda galaxy (Fardal et al. 2008, 2012). Moreover, the age of the stellar populations believed to drive HI supershells are far too young to have been affected by a satellite disk passage (e.g., akin to the situation observed in NGC 5387; Beaton et al. 2014). Assuming a projected radius for the stream of $\sim 40-50 \mathrm{kpc}$, it would be unlikely that the progenitor would pass through a gas-rich portion of the NGC 4631 disk. Thus, we suspect that neither the gas streams nor the star forming regions are associated with the stellar tidal 
stream. It is worth noting that ram pressure stripping of the HI gas could be a contributing factor to explain why the two components of the stellar stream and the HI features, particularly in respect to the stream $\mathrm{SE}$, do not line up with each other.

\section{PHOTOMETRIC PROPERTIES AND STELLAR MASS LIMITS OF THE STREAM}

Our analysis primarily focuses on the two most extended substructures in the NGC 4631 stellar halo-stream ${ }_{\mathrm{NW}}$ (which possibly includes the progenitor of the stream) and stream ${ }_{\mathrm{SE}}$ as identified in Figure 3. Using the calibrated ROSA luminance image, we construct isophotes for each of the features corresponding to the $5 \sigma$ large-scale background variation within the $L$ data (i.e., the $\mu_{\mathrm{L}}=27.7 \mathrm{mag} \operatorname{arcsec}^{-2}$ isophote). In Figure 3, these photometric boundaries are displayed for the features of interest. Due to $\mathrm{S} / \mathrm{N}$ limitations of our data, there may be faint emission associated with these features that lies outside of these boundaries. Therefore, the luminosities we report hereafter are lower limits.

For both stream components, we have measured the average surface brightness within the isophotal boundaries. For stream $_{\mathrm{NW}}$, we measure $\left\langle\mu_{\mathrm{L}}\right\rangle=27.0 \pm 0.1 \mathrm{mag} \operatorname{arcsec}^{-2}$. With $\left\langle\mu_{\mathrm{L}}\right\rangle=27.4 \pm 0.2 \mathrm{mag} \mathrm{arcsec}^{-2}$, stream $_{\mathrm{SE}}$ is thus marginally fainter than the measurement uncertainties. For comparison, the average Luminance surface brightness within the isophotal boundaries of DW1 and DW2 are $27.0 \pm 0.1 \mathrm{mag} \mathrm{arcsec}^{-2}$ and $26.45 \pm 0.07 \mathrm{mag} \operatorname{arcsec}^{-2}$, respectively (but note that the average surface brightness measurement of DW2 may be contaminated by light from the outskirts of the NGC 4631 disk). To measure the PA of each of the two stream components and to aid in the measurement of their widths (see below), ellipses were fit to the $\mu_{\mathrm{L}}=27.7 \mathrm{mag} \mathrm{arcsec}^{-2}$ isophotal boundary of each stream component. As mentioned in Section 3, the major axes of these fitted ellipses each have a measured PA of $136^{\circ}$.

Assuming that stream $_{\mathrm{NW}}$ and stream $_{\mathrm{SE}}$ have a common origin and are at the same distance of NGC 4631, the total projected length of that structure is $\geqslant 85.2 \mathrm{kpc}$, as measured from the far edges of the ellipses fit to the isophotal boundaries of each of the two stream components. We also measured the widths of stream $\mathrm{SE}$ and the possible stream progenitor (within stream $\left._{\mathrm{NW}}\right)$. To make this measurement for each stream component, we collapse a strip of the image using a median combine (excluding pixels that are masked due to foreground stellar sources) along the direction of the fitted ellipse's major axis (see the dashed green lines in Figure 3). The result is a flux profile along the minor axis of the isophote for each object on which the measurement strip is centered. The strip is $\pm 90^{\prime \prime}$ $( \pm 3.23 \mathrm{kpc})$ in width about the minor axis of each isophotal boundary. The widths are measured by fitting a Gaussian function to each light profile, from which we obtain a FWHM of $4.69 \pm 0.03$ and $2.53 \pm 0.02 \mathrm{kpc}$ for stream $_{\mathrm{SE}}$ and the progenitor candidate (within stream $\mathrm{NW}_{\mathrm{W}}$ ), respectively. Note that the quoted uncertainties in the FWHM do not include the systematic uncertainty associated with the adopted distance to NGC 4631. As indicated by these measurements and illustrated in Figure 5(a), stream $\mathrm{SE}_{\mathrm{SE}}$ is wider than stream $_{\mathrm{NW}}$ by a factor of $\sim 2$. Figure 5(b) shows the width of each stream component as a function of distance along the major axis of their isophotal boundaries, illustrating that the width of stream $_{\mathrm{NW}}$ is consistently narrower than that of stream ${ }_{\mathrm{SE}}$, even away from its bright core. These width measurements were performed in the same way as those shown in Figure 5(a), except with nonoverlapping individual measurement strips that were $62^{\prime \prime}$ $(2.22 \mathrm{kpc})$ in width. In Figure $5(\mathrm{~b})$, the decreasing distance along the major axis indicates positions that are closer to NGC 4631.

To estimate a stellar mass for stream ${ }_{\mathrm{NW}}$ and stream $_{\mathrm{SE}}$, we must obtain a measurement of the total luminosity from our calibrated luminance image $\left(L_{\mathrm{L}}\right)$ and an estimate of the stellar mass-to-light ratio over that same passband $\Upsilon_{L}$. To calculate the former, we measure the total flux within the isophotal boundaries. Note that pixels within the boundary that are masked for foreground stellar sources are replaced by averaging linearly interpolated values along pixel rows and columns. Taking into account the adopted distance to NGC 4631 and recalling that additional faint emission may lie outside of the boundaries, the measured fluxes correspond to lower limits on the luminance-band stellar luminosity of $1.80 \times 10^{7} L_{\odot, \mathrm{L}}$ for stream $_{\mathrm{NW}}$ and $1.46 \times 10^{7} L_{\odot, \mathrm{L}}$ for the stream $_{\mathrm{SE}}$.

As opposed to obtaining the total luminosity of the stream components within their isophotal boundaries, an estimation of $\Upsilon_{\mathrm{L}}$ is less trivial since it requires knowledge of the color of the stellar populations (e.g., Zibetti et al. 2009). Unfortunately, the ROSA data do not provide this color information. For the purposes of this discussion, we assume a red color for the two stream components that is consistent with the colors of a typical S0 galaxy (Barway et al. 2005) and the redder dwarf galaxies in the Local Group (Mateo 1998). This assumption is consistent with measurements of the color of stellar streams around NGC $4013\left(B-R=1.6_{-0.4}^{+0.6}\right.$; Martínez-Delgado et al. 2009) and M63 ( $B-R=1.5 \pm 0.2$; Chonis et al. 2011). To transform these representative $B-R$ colors into a $g-r$ color that is consistent with the photometric system utilized here to calibrate our luminance-filter observations, we use the data set from Chonis \& Gaskell (2008) that was used to derive UBVRI to ugriz photometric transformation equations. We find that $(g-r)=0.685 \pm 0.015(B-R)-0.246 \pm 0.018$. Thus, a $B-R$ color of 1.5 , which is consistent with previously measured stellar streams around nearby spiral galaxies, corresponds to a $g-r$ color of approximately 0.8 .

We utilize the relations giving the mass-to-light ratio in different passbands as a function of various color indexes from Zibetti et al. (2009), who assume a Chabrier (2003) initial mass function. For the $g-r$ color index using the weighting coefficient $c_{2}$ for the SDSS $g$ and $r$-bands from Section 2, we estimate the Luminance mass-to-light ratio as:

$$
\Upsilon_{\mathrm{L}}=c_{2} \Upsilon_{g}(g-r)+\left(1-c_{2}\right) \Upsilon_{r}(g-r)
$$

where $\Upsilon_{g}(g-r)=10^{-1.030+2.053(g-r)} \quad$ and $\quad \Upsilon_{r}(g-r)=$ $10^{-0.840+1.654(g-r)}$. For an assumed $g-r$ color of 0.8 , this estimation yields $\Upsilon_{\mathrm{L}}=3.36$ for the stream components. Combining this mass-to-light ratio estimate and the lower limit on the total luminosity of each stream component, we find stellar mass $M_{*}$ lower limits of $6.04 \times 10^{7} M_{\odot}$ for stream $_{\mathrm{NW}}$

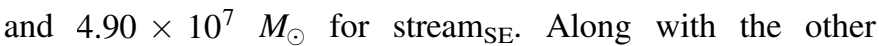
photometrically derived properties of the two stream components, these stellar mass limits are tabulated in Table 1. Assuming that both features are part of the same large tidal 

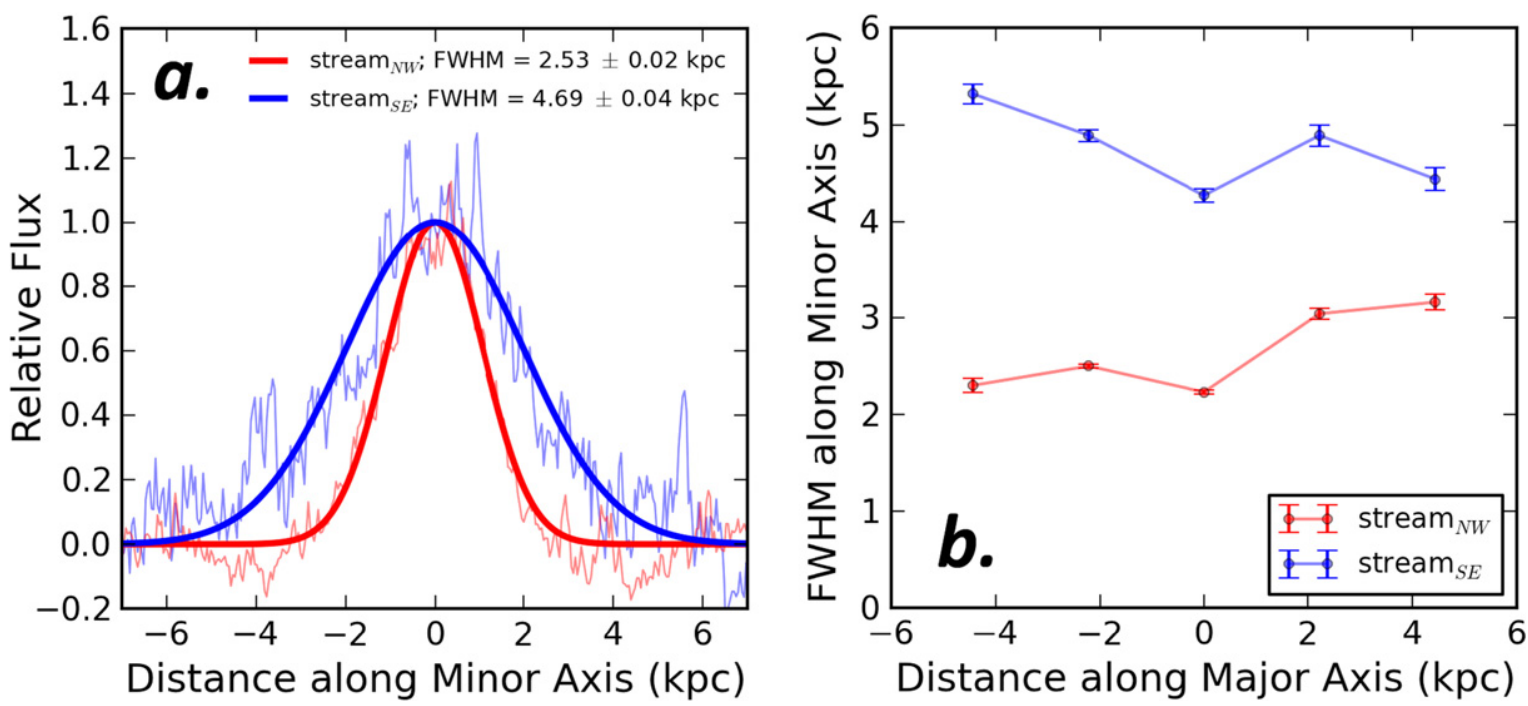

Figure 5. Measurements of the widths of stream $\mathrm{SE}_{\mathrm{SE}}$ and stream $\mathrm{NW}_{\mathrm{NW}}$. (a) Light profiles along the minor axis of the isophotes for each stream component measured over a $\pm 90^{\prime \prime}( \pm 3.23 \mathrm{kpc})$ strip. The colored light curves show the median combined light profiles, while the heavy curves show the best-fit Gaussians whose widths and statistical uncertainties are shown at the top of the plot. The flux profiles are shown normalized to the Gaussian peaks to facilitate visual comparison. (b) The FWHM of each stream component measured parallel to the minor axis of their isophotal boundaries as a function of distance along the major axis. Each data point corresponds to the FWHM of a Gaussian fit to the median light profile measured over a 62" $(2.22 \mathrm{kpc})$ wide, non-overlapping strip. Decreasing distance along the major axis corresponds decreasing radial distance from NGC 4631.

Table 1

Surface Photometry of Sub-structure Around NGC 4631

\begin{tabular}{lccc}
\hline \hline & & Stream $_{\mathrm{NW}}$ & Stream $_{\mathrm{SE}}$ \\
\hline$A^{\mathrm{a}}$ & $\operatorname{arcsec}^{2}$ & 20484 & 24383 \\
$\mathrm{PA}^{\mathrm{b}}$ & $\circ$ & 136 & 136 \\
$\left\langle\mu_{\mathrm{L}}\right\rangle^{\mathrm{c}}$ & $\left(\mathrm{mag} \mathrm{arcsec}^{-2}\right)$ & $27.0 \pm 0.1$ & $27.4 \pm 0.2$ \\
$\mathrm{FWHM}^{\mathrm{d}}$ & $(\mathrm{kpc})$ & $2.53 \pm 0.02$ & $4.69 \pm 0.03$ \\
$L_{\mathrm{L}}{ }^{\mathrm{e}}$ & $\left(L_{\odot, \mathrm{L}}\right)$ & $>1.80 \times 10^{7}$ & $>1.46 \times 10^{7}$ \\
$M_{*}{ }^{\mathrm{f}}$ & $\left(M_{\odot}\right)$ & $>6.04 \times 10^{7}$ & $>4.90 \times 10^{7}$ \\
\hline
\end{tabular}

Notes.

${ }^{\text {a }}$ Area enclosed within the $\mu_{\mathrm{L}}=27.7 \mathrm{mag} \operatorname{arcsec}^{-2}$ isophote.

${ }^{b}$ Position angle of the major axis of an ellipse fit to the $\mu_{\mathrm{L}}=27.7$ mag $\operatorname{arcsec}^{-2}$ isophote.

${ }^{\mathrm{c}}$ Mean surface brightness averaged over the area $A$.

${ }^{\mathrm{d}}$ The quoted uncertainties are statistical and do not include the systematic uncertainty in the adopted distance to NGC 4631.

${ }^{\mathrm{e}}$ Lower limits calculated through measurements of the total flux contained within the area $A$.

${ }^{\mathrm{f}}$ Lower limits calculated assuming the integrated color of the stream is $g-r=0.8$.

structure, the lower limit on its total stellar mass is $1.09 \times 10^{8}$ $M_{\odot}$.

To obtain a better handle on the stellar mass-to-light ratios of these features, deep multi-band surface photometry is required. In addition, such data would be highly valuable for finding color gradients within or among the stream's components. For example, if the color of stream $\mathrm{NW}_{\mathrm{W}}$ was found to be bluer than stream $_{\mathrm{SE}}$, additional strong emperical arguments could be made to further support the hypothesis that stream $_{\mathrm{NW}}$ contains the stream progenitor.

\section{A MODEL FOR THE STELLAR STREAM}

The aim of this section is to explore whether the observed stream-like feature around NGC 4631 can be directly linked to interactions between this spiral galaxy and one of its dwarf companions by means of a theoretical model. We also note, as previously demonstrated by Toomre \& Toomre (1972) and D'Onghia et al. (2010), that tidal tails and bridges connecting galaxy pairs continue both to lengthen and to thin out after the encounter. It was shown in these studies that the efficiency of tail formation depends on the inclinations of the disks relative to the orbital plane. NGC 4656 and NGC 4631 are both almost edge-on (e.g., see Figure 2) and, in particular, NGC 4656 is inclined almost $90^{\circ}$ relative to the orbital plane of NGC 4631. This configuration inhibits the formation of any tail or stellar stream and excludes that any effective tidal interaction between NGC 4656 and NGC 4631 is responsible of the observed extended structure.

In our numerical experiment, we only chose to focus on the extended stellar stream around NGC 4631. Thus, the goal is not to reproduce every detail of this complex system-the interactions of the numerous companions, the HI streams, and the stellar streams-but rather to produce a viable model for the feature reported here. A model incorporating all of the observed debris features in the NGC 4631 halo and its interaction with NGC 4565 would require a parameter search of all the possible orbital configurations and mass ratios for each of the galaxies, which is beyond the scope of the work presented here.

We follow the general method outlined in D'Onghia et al. (2009) to set up the initial galaxy models and satellite orbits to reproduce the observed large-scale stellar stream around NGC 4631. In the model, NGC 4631 consists of a dark matter halo and a rotationally supported stellar disk. The parameters describing each component are independent and the models are constructed in a manner similar to the approach presented in previous works. We model the dark matter mass distribution of each galaxy with a Hernquist (1990) profile and an exponential stellar disk. The total halo mass of NGC 4631 is assumed to be $M_{\text {halo }}=4 \times 10^{11} M_{\odot}$ and a halo scale length of $22 \mathrm{kpc}$. The disk is a particle-realization of an exponential disk with a constant scale-height, in which both the positions and velocities of the particles are assigned following the prescriptions in Springel 


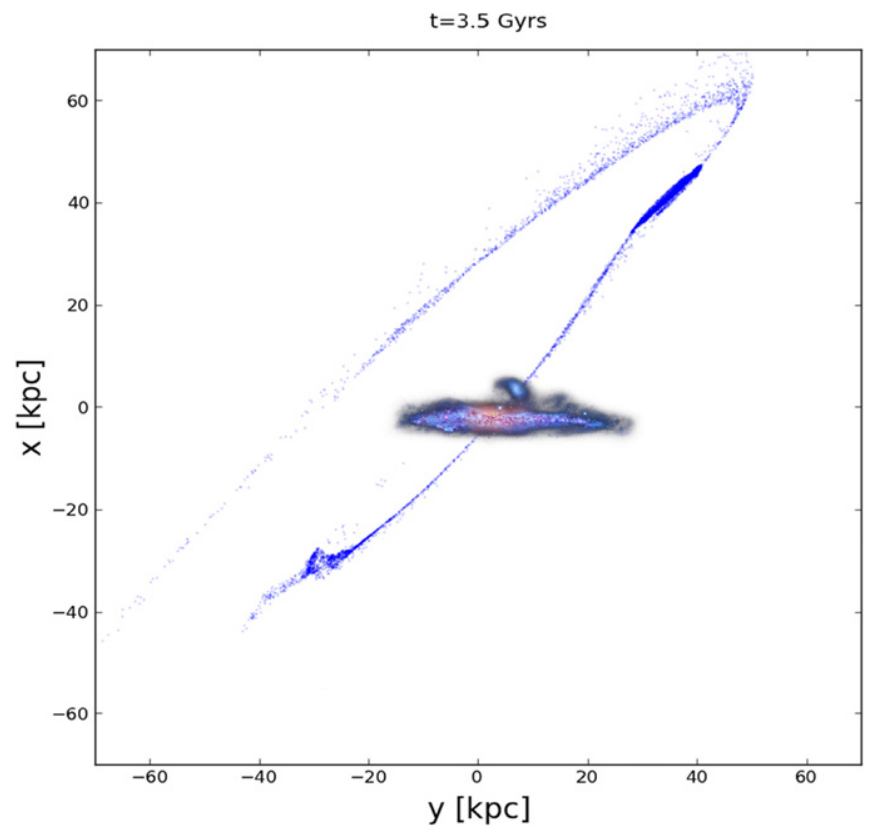

Figure 6. Snapshot of the $N$-body simulation that qualitatively reproduces both stream features in NGC 4631 from an interaction between it and a single dwarf satellite on a mildly eccentric orbit $(e=0.6)$. The stream is displayed at $t \sim$ $3.5 \mathrm{Gyr}$ into the interaction. Only a single interaction, the one creating the stellar stream, is modeled. Owing to the numerous dwarf companions and tidal debris observed in NGC 4631, a far more complex model would be required to reproduce all of the features and is beyond the scope of this work.

et al. (2005). We adopt a radial scale length for the disk of $R_{\text {disk }}=2 \mathrm{kpc}$, with a scale-height given by $z_{0}=0.1 R_{\text {disk }}$. The rotation curve of NGC 4631 peaks at $130 \mathrm{~km} \mathrm{~s}^{-1}$ in agreement with observational estimates (Combes et al. 1977, and references therein).

We model a dwarf satellite galaxy consisting of a dark matter halo of $10^{10} M_{\odot}$, a scale length of $9 \mathrm{kpc}$ and a stellar mass fraction of $4 \%$ of the total mass. The number of particles of each component $\left(N_{*}\right.$ for the number of star particles stars, $N_{\text {halo }}$ for dark matter particles) is chosen such that the mass resolution per particle of a given type is roughly the same for both galaxies. We adopt $N_{\text {halo }}=10^{5} ; 10^{4}$ and $N_{*}=10^{6} ; 10^{5}$ to model NGC 4631 and its orbiting dwarf, respectively.

Generally, higher orbital eccentricities for the satellite result in "fly-by" encounters, while lower values cause the dwarf's orbit to decay rapidly. Given the morphology of the debris, the dwarf galaxy is placed on an eccentric orbit about NGC 4631 $(e=0.6)$. With this given orbit, the pericentric distance of the dwarf from the center of NGC 4631 is around $R_{\text {peri }}=15 \mathrm{kpc}$ and the apocentric distance is $R_{\text {apo }}=60 \mathrm{kpc}$.

The satellite is placed in an orbit around NGC 4631 and is evolved over a period of $\sim 3.5 \mathrm{Gyr}$. The simulations were carried out with GADGET3, a parallel TreePM-Smoothed particle hydrodynamics code developed to compute the evolution of stars and dark matter, both of which are treated as collisionless fluids.

Figure 6 displays the resulting $N$-body model showing the density of star particles at $t \sim 3.5$ Gyr. During the simulations, stars are pulled out of the dwarf satellite in its orbit around NGC 4631 to form a long stream that is in qualitative agreement with the observed stream displayed in Figures 2 and 3 . A closer inspection shows that the angular thicknesses of the stream do not perfectly match the component of the stream labeled stream $\mathrm{SE}_{\mathrm{SE}}$. The match might improve if the progenitor were more massive. However, given the quality of the data presented in this work it is hard to find a conclusive answer. Further comparisons will be done when more detailed data are available for this system.

In the model, what remains of the dwarf is contained within the simulated analog of the stream $_{\mathrm{NW}}$ feature. Assuming that our derived stream stellar mass lower limit $\left(1.09 \times 10^{8} M_{\odot}\right.$; see Section 4 ) is $\sim 20 \%$ of the total mass of the progenitor, as suggested by simulations of merging galaxies (Cox et al. 2006), our simulations suggest a total initial stellar mass of $5.4 \times 10^{8} M_{\odot}$ for the dwarf and a total dynamical mass (including dark matter) of $1.36 \times 10^{10} M_{\odot}$. Thus the mass ratio of the interaction between NGC 4631 and the dwarf satellite causing the observed stellar stream is approximately 25-30.

\section{CONCLUSIONS}

We report the discovery of an extended stellar tidal stream around NGC 4631. From our data, combined with arguments based on simulations of tidal interactions, we conclude that this newly detected stellar stream cannot be due to interactions between NGC 4631 and the galaxy NGC 4656. Intriguingly, the discovered stellar stream lies between previously detected HI streams (referred to as spurs 1 and 2), which indicates that the stellar stream is most likely not related to these features and thus produced by a different progenitor satellite. This stellar substructure is added to an already rich census of substructure in the NGC 4631 halo, including five HI "spurs" with overall structure very similar to that of the Magellanic Stream in the MW halo, and to two HI supershells associated with massive star-forming regions in its disk. Thus, the NGC 4631 stellar halo contains numerous on-going accretion events, akin to the complexity observed in the stellar halos of the Local Group spirals.

The feature observed around NGC 4631 is reproduced by numerical simulations of the tidal interaction of a candidate dwarf galaxy (embedded in stream ${ }_{\mathrm{NW}}$ ) with its parent, NGC 4631. The model qualitatively agrees with our assessment from the optical morphology of the features.

Alternative progenitors for the stream, like NGC 4627, seem to be unlikely given its orientation with respect both streams. Radial velocity measurements and more detailed observations of the stream will be able to discriminate between the different scenarios and will be explored in a future work.

We recovered two previously detected faint dwarf galaxy candidates associated with the NGC 4631 system that could be the progenitors of the gas streams. For example, DW1 might be associated with spur 1 and a possible candidate DW2 could be associated with spur 4. However, the investigation of the full complement of dwarf satellite interactions and debris features within the NGC 4631 halo is beyond the scope of this paper and it will be explored in a forthcoming study.

A visual inspection of the projected positions of dwarf candidates around NGC 4631 and the path of the stellar stream might suggest that the substructures might be aligned within an extended plane. Similar configurations have been claimed to have been found among satellite galaxies around M31 (Koch \& Grebel 2006; Ibata et al. 2014) and our MW (e.g., Pawlowski et al. 2012). It has been suggested that this confinement of satellite galaxies in thin planes around galaxies presents a challenge to the theory of hierarchical galaxy clustering (D’Onghia \& Lake 2008; Pawlowski et al. 2014) but a number 
of recent simulations show that such structures are expected in $\Lambda$ CDM (e.g., Libeskind et al. 2015; Tempel et al. 2015). In this context, NGC 4631 and its complex system of satellite galaxies represent an additional interesting case to study the problem of how these satellites fell into a more massive system. However, follow-up observations to derive accurate distances (by means of its resolved stellar population) and radial velocities are needed to shed light on the actual spatial configuration of these structures and their possible common origin.

We thank the anonymous referee for useful comments and suggestions that have improved this paper. We thank R. Rand for the use of his VLA HI data cube and M. Donahue for a physical copy of her 1995 paper for comparison to our new image. D. M. D. and E. K. G. acknowledge funding from Sonderforschungsbereich SFB 881 "The Milky Way System" (subproject A2) of the German Research Foundation (DFG). E. D. acknowledges support of the Alfred P. Sloan Foundation and Grant No. AST1211258 and ATP-NASA Grant No. NX14AP53G. The simulation has been run on the High Performance Computing cluster provided by the Advanced Computing Infrastructure (ACI) and Center for High Throughput Computing (CHTC) at the University of Wisconsin. E. D. and R. L. B. express their appreciation toward the Aspen Center for Physics and the NSF Grant N. PHYS-1066293 for hospitality during the writing of this paper. T. S. C. acknowledges the support of the National Science Foundation Graduate Research Fellowship. Funding for SDSS-III has been provided by the Alfred P. Sloan Foundation, the Participating Institutions, the National Science Foundation, and the U.S. Department of Energy Office of Science. The SDSS-III web site is http://www.sdss3.org/. SDSS-III is managed by the Astrophysical Research Consortium for the Participating Institutions of the SDSS-III Collaboration including the University of Arizona, the Brazilian Participation Group, Brookhaven National Laboratory, Carnegie Mellon University, University of Florida, the French Participation Group, the German Participation Group, Harvard University, the Instituto de Astrofisica de Canarias, the Michigan State/Notre Dame/JINA Participation Group, Johns Hopkins University, Lawrence Berkeley National Laboratory, Max Planck Institute for Astrophysics, Max Planck Institute for Extraterrestrial Physics, New Mexico State University, New York University, Ohio State University, Pennsylvania State University, University of Portsmouth, Princeton University, the Spanish Participation Group, University of Tokyo, University of Utah, Vanderbilt University, University of Virginia, University of Washington, and Yale University.

\section{REFERENCES}

Abazajian, K. N., Adelman-McCarthy, J. K., Agüeros, M. A., et al. 2009, ApJS, 182, 543
Abraham, R. G., \& van Dokkum, P. G. 2014, PASP, 126, 55

Ann, H. B., Seo, M. S., \& Baek, S.-J. 2011, JKAS, 44, 23

Arp, H. 1966, ApJS, 14, 1

Barway, S., Mayya, Y. D., Kembhavi, A. K., \& Pandey, S. K. 2005, AJ, 129,630

Beaton, R. L., Martínez-Delgado, D., Majewski, S. R., et al. 2014, ApJ, 790, 117

Belokurov, V., Zucker, D. B., Evans, N. W., et al. 2006, ApJL, 642, L137

Bullock, J. S., \& Johnston, K. V. 2005, ApJ, 635, 931

Chabrier, G. 2003, PASP, 115, 763

Chonis, T. S., \& Gaskell, C. M. 2008, AJ, 135, 264

Chonis, T. S., Martínez-Delgado, D., Gabany, R. J., et al. 2011, AJ, 142, 166 Combes, F. 1978, A\&A, 65, 47

Combes, F., Encrenaz, P. J., Lucas, R., \& Weliachew, L. 1977, A\&A, 55, 311 Cooper, A. P., Cole, S., Frenk, C. S., et al. 2010, MNRAS, 406, 744

Cox, T. J., Suvendra, N., di Matteo, T., et al. 2006, ApJ, 650, 791

Donahue, M., Aldering, G., \& Stocke, J. T. 1995, ApJL, 450, L45

D’Onghia, E., Besla, G., Cox, T. J., \& Hernquist, L. 2009, Natur, 460, 605

D’Onghia, E., \& Lake, G. 2008, ApJL, 686, L61

D’Onghia, E., Springel, V., Hernquist, L., \& Keres, D. 2010, ApJ, 709, 1138

Duc, P.-A., Cuillandre, J.-C., Kurabal, E., et al. 2014, MNRAS, 446, 120

Fardal, M. A., Babul, A., Guhathakurta, P., Gilbert, K. M., \& Dodge, C. 2008, ApJL, 682, L33

Fardal, M. A., Guhathakurta, P., Gilbert, K. M., et al. 2012, MNRAS, 423, 3134

Foster, C., Lux, H., Romanowsky, A. J., et al. 2014, MNRAS, 442, 3544

Hernquist, L. 1990, ApJ, 356, 359

Ibata, R. A., Ibata, N. G., Lewis, G. P., et al. 2014, ApJ, 784, 6

Johnston, K. V., Bullock, J. S., Sharma, S., et al. 2008, ApJ, 689, 936

Karachentsev, I. D., Bautzmann, D., Neyer, F., et al. 2014, arXiv:1401.2719

Koch, A., \& Grebel, E. K. 2006, AJ, 131, 1405

Libeskind, N. I., Hoffman, Y., Tully, R. B., et al. 2015, MNRAS, in press (arXiv:1503.05915)

Ludwig, J., Pasquali, A., Grebel, E. K., \& Gallagher, J. S., III 2012, AJ, 144,190

Majewski, S. R., Skrutskie, M. F., Weinberg, M. D., \& Ostheimer, J. C. 2003, ApJ, 599, 1082

Martínez-Delgado, D., Gabany, R. J., Crawford, K., et al. 2010, AJ, 140, 962 Martínez-Delgado, D., Pohlen, M., Gabany, R. J., et al. 2009, ApJ, 692, 955 Mateo, M. 1998, ARA\&A, 36, 435

McConnachie, A. W., Irwin, M. J., Ibata, R. A., et al. 2009, Natur, 461, 66

Miskolczi, A., Bomans, D. J., \& Dettmar, R.-J. 2011, A\&A, 536, A66

Mutch, S. J., Croton, D. J., \& Poole, G. B. 2011, ApJ, 736, 84

Odenkirchen, M., Grebel, E. K., Rockosi, C. M., et al. 2001, ApJ, 548, 165

Paudel, S., Duc, P.-A., Côté, P., et al. 2013, ApJ, 767, 133

Pawlowski, M. S., Pflamm-Altenburg, J., \& Kroupa, P. 2012, MNRAS, 423, 1109

Pawlowski, M. S., Famaey, B., Jerjen, H., et al. 2014, MNRAS, 442, 2362

Pillepich, A., Madau, P., \& Mayer, L. 2014, arXiv:1407.7855

Radburn-Smith, D. J., de Jong, R. S., Seth, A. C., et al. 2011, ApJS, 195, 18

Rand, R. J. 1994, A\&A, 285, 833

Rand, R. J., \& van der Hulst, J. M. 1993, AJ, 105, 2098

Rots, A. H. 1978, AJ, 83, 219

Schechtman-Rook, A., \& Hess, K. M. 2012, ApJ, 750, 171

Schlafly, E. F., \& Finkbeiner, D. P. 2011, ApJ, 737, 103

Schlegel, D. J., Finkbeiner, D. P., \& Davis, M. 1998, ApJ, 500, 525

Schweizer, F., \& Seitzer, P. 1988, ApJ, 328, 88

Slater, C. T., Bell, E. F., Schlafly, E. F., et al. 2014, ApJ, 791, 9

Springel, V., White, S. D. M., Jenkins, A., et al. 2005, Natur, 435, 629

Tempel, E., Guo, Q., Kipper, R., \& Libeskind, N. I. 2015, MNRAS, 450, 2727

Toomre, A., \& Toomre, J. 1972, ApJ, 178, 623

Zibetti, S., Charlot, S., \& Rix, H.-W. 2009, MNRAS, 400, 1181 